\title{
Enzalutamide: A Step Towards Pharmacokinetic-Based Dosing in Men with Metastatic Castration-Resistant Prostate Cancer
}

\author{
Paul R. Hutson ${ }^{1}$
}

Published online: 16 June 2015

(c) Springer International Publishing Switzerland 2015

Other than skin cancer, prostate cancer is the most commonly diagnosed cancer in men, and although usually curable when diagnosed and treated early, it remains the second most common cause of cancer-related death in the US among men [1]. Treatment of low-stage, limited disease with radiation or prostatectomy is associated with a high long-term cure rate. 'Watchful waiting' monitoring or active surveillance of low-stage, low-grade prostate cancer is considered appropriate and delays the treatment-related side effects of incontinence and impotence. Progressive or advancing local cancer limited to the prostatic capsule may still be curable but this is not the case when prostate cancer metastasizes outside the prostate. The most common sites of prostate cancer metastases are the spinal vertebra, liver, and lung.

Prostate cancer is a hormone-sensitive disease, therefore the treatment of metastatic disease usually focuses on the removal of androgenic stimulation. This can be done with surgical or medical castration. Medical castration is usually performed using a luteinizing hormone-releasing hormone (LHRH) agonist such as leuprolide that indirectly decreases testicular testosterone production. The goal is to lower testosterone concentrations to $<50 \mathrm{ng} / \mathrm{dl}(1.73 \mathrm{nM})$.

At some point, prostate cancer may lose its dependence on testosterone for proliferation and, if metastasized beyond the prostate capsule, is termed metastatic,

This comment refers to the articles available at doi:10.1007/s40262015-0271-5 and 10.1007/s40262-015-0283-1.

Paul R. Hutson

paul.hutson@wisc.edu

1 University of Wisconsin School of Pharmacy, 777 Highland Avenue, Madison, WI, USA castration-resistant prostate carcinoma (mCRPC). The mechanism of this resistance to androgen deprivation can be multifactorial, but most commonly it is thought to arise from the autocrine or paracrine production of testosterone or other androgens, such as dehydroepiandrostenedione (DHEA), which stimulate growth of the cancer. Several treatment approaches exist for the treatment of mCRPC, including cytotoxic treatment with docetaxel plus prednisone, inhibitors of androgen synthesis such as abiraterone acetate plus prednisone, or androgen inhibitors such as enzalutamide. The current standard of treatment for mCRPC is treatment with docetaxel plus prednisone; however, recent trials suggest that the use of androgen synthesis inhibitors or receptor antagonists can delay the need to start the more toxic chemotherapy.

Enzalutamide is a second-generation antagonist of the androgen receptor which appears to have a more consistent effect than bicalutamide, which may, over time, develop paradoxical agonist activity [2, 3]. Enzalutamide was shown to have activity in slowing the progression of cancer growth in patients who had progressed on docetaxel plus prednisone in the AFFIRM (Atrial Fibrillation Follow-up Investigation of Rhythm Management) trial [4]. More recently, it was also shown to slow the progression of prostate cancer and delay the need for docetaxel plus prednisone in the PREVAIL trial [5]. In both trials, the response to enzalutamide versus placebo was so remarkable that the studies were closed after the first planned interim analysis.

Enzalutamide acts by binding to the ligand binding domain (LBD) of the androgen receptor, blocking the ability of androgen agonists to bind to the site. Additionally, enzalutamide inhibits the ability of the activated androgen receptor to translocate to the nucleus and to bind 
to the transcription binding domain of DNA. Resistance to the effect of enzalutamide appears to occur most commonly through mutations in the LBD that limit the ability of the drug to bind to the site [6]. Other androgen receptor antagonists that bind to other sites on the receptor separate from the LBD are under clinical investigation. Current clinical trials of enzalutamide include those that are evaluating the benefit of combining the drug with the antiprogestin mifepristone, or with a rapid cycling of exogenous testosterone in patients progressing through androgen ablation and enzalutamide.

This issue of Clinical Pharmacokinetics presents two reports from the sponsoring pharmaceutical companies and their distinguished clinician collaborators, detailing the clinical pharmacokinetics of enzalutamide [7, 8]. Notable and appreciated is the article that combines the findings of five separate pharmacokinetic studies into one report. This consolidation leads to a very readable and efficient presentation of their findings. In addition to noncompartmental methods, the authors apply modeling with nonlinear mixed-effects methods (NONMEM) to describe the pharmacokinetics of the drug from single doses, regular dosing from phase III trials, and from subjects with varying degrees of hepatic failure.

Enzalutamide is known to be metabolized to the $N$-desmethyl enzalutamide that has plasma concentrations and activity similar to that of the parent compound, and to a carboxylic acid metabolite that is thought to be inactive. The dose of enzalutamide $160 \mathrm{mg}$ used in the clinical trials was chosen due to no clear improvement of response with higher doses and an increased incidence of adverse events, including seizures [3]. Gibbons and colleagues demonstrate the expected accumulation of a drug with a 6-day terminal elimination half-life administered daily [7]. Their findings also demonstrate an even longer elimination half-life of the active $N$-desmethyl metabolite. Neither the area under the concentration-time curve (AUC) and maximum concentration $\left(C_{\max }\right)$ of the parent or the $N$-desmethyl metabolite were found to be substantially affected by hepatic failure categorized as mild to moderate. This may lead to preferred use of enzalutamide in men with prostate cancer metastatic to the liver, instead of docetaxel, which is known to have a more substantial inhibition of its clearance in mild to moderate liver disease. Similarly, food did not have a substantial effect on the pharmacokinetics of the parent or $\mathrm{N}$-desmethyl enzalutamide exposure, simplifying subject dosing.

The variability of enzalutamide and its $N$-desmethyl metabolite $C_{\max }$ and AUC among subjects was low in the single-dose studies, and the exposure to active drug (parent plus $N$-desmethyl metabolite) did not appear to affect the overall survival of subjects when high versus low trough concentrations $\left(C_{\text {trough }}\right)$ quartiles were compared. Rather than suggesting a flat concentration versus effect curve, the lack of an exposure effect may be due to low variability in the $C_{\text {trough }}$ values among the population, since the ratio of the medians of the top and bottom quartiles of $C_{\text {trough }}$ was only 1.5 .

Gibbons et al. also provide a very useful and cleverly designed clinical trial evaluating the effect of enzalutamide on the activity of multiple cytochrome P450 (CYP) isozymes using a substrate cocktail for CYP3A4 (midazolam), CYP2C8 (pioglitazone), CYP2C9 (warfarin), and CYPC19 (omeprazole) [8]. Furthermore, they assess the effect of inhibitors of CYP3A4 (itraconazole) and CYP2C8 (gemfibrozil) upon the pharmacokinetics of enzalutamide and its $N$-desmethyl and carboxy metabolites. In contrast to in vitro studies in the original prescribing information [9], which suggested that the drug is an inhibitor of these CYP isoforms as well as 2B6 and 2D6, the present clinical study shows the opposite effect, with induction by enzalutamide of CYP2C9, 2C19 and, in particular, 3A4. The current drug interaction study apparently did not evaluate the effect of enzalutamide upon the activity of CYP2B6 or 2D6, and it is hoped that the effect upon these two important isoforms will be studied in the near future in order to guide therapy.

Mentioned briefly in their discussion, it is appropriate to emphasize that the induction of CYP3A4 by enzalutamide suggests that it may also induce the activity of glucuronidation and transporters such as P-glycoprotein (Pgp). Induction of glucuronidation is suggested by the substantial lowering of the AUC of the metabolites of the P450 probe drugs, when higher metabolite AUCs would be expected from enzalutamide induction if no induction of downstream metabolizing enzymes also occurred. This is of substantial importance as enzalutamide is tested in the future with androgen synthesis inhibitors such as the CYP3A4 substrate abiraterone acetate, as well as other drugs taken by men for comorbidities. One of the important implications of the very long terminal half-lives of enzalutamide and its metabolites is that the induction of CYP3A4, 2C9, and 2C19, and possibly of glucuronidation or transporters, can be expected to persist for weeks after the cessation of treatment. This will affect the exposure to drugs such as docetaxel or abiraterone which may be chosen to replace enzalutamide in the presence of progressive disease.

Gibbons et al. do not report the renal clearance or the effect of renal failure on the clearance of enzalutamide or its active $N$-desmethyl metabolite, and to date no other reports of enzalutamide renal clearance are available to guide dosing. The mass-balance studies in the present study demonstrate that approximately $60 \%$ of the dose was recovered in the urine, suggesting that dosing of the drug may need to be reduced in patients with kidney dysfunction. In addition to reports of the renal clearance of enzalutamide, it is important for studies to be performed to 
determine whether there is a clinically significant effect of enzalutamide treatment on transporter activity or that of glucuronidation.

Despite some wishlist items mentioned, in these two well-executed and written studies, Gibbons and colleagues have provided a plethora of practical information to guide the future use of enzalutamide in men with prostate cancer. The consolidation of multiple smaller studies adds to the utility of these reports, and should be encouraged of other investigators.

Disclosures No sources of funding were used in the assistance of this commentary. Paul R. Hutson has no conflicts of interest to declare.

\section{References}

1. National Cancer Institute, Surveillance, Epidemiology, and end results program. Fast Stats. Available at: http://Seer.cancer.gov/ faststats. Accessed 25 May 2015.
2. Tran C, Ouk S, Clegg NJ, et al. Development of a secondgeneration antiandrogen for treatment of advanced prostate cancer. Science. 2009;324:787-90.

3. Higano CS, Beer TM, Taplin ME, et al. Long-term safety and antitumor activity in the phase 1-2 study of enzalutamide in preand post-docetaxel castration-resistant prostate cancer. Eur Urol. 2015. doi:10.1016/j.eururo.2015.01.026 (Epub 16 Feb).

4. Scher HI, Fizazi K, Saad F, et al. Increased survival with enzalutamide in prostate cancer after chemotherapy. N Engl J Med. 2012;367:1187-97.

5. Beer TM, Armstsrong AJ, Rathkopf DE, et al. Enzalutamide in metastatic prostate cancer before chemotherapy. N Engl J Med. 2014;371:424-33.

6. Karantanos T, Evans CP, Tombal B, et al. Understanding the mechanisms of androgen deprivation resistance in prostate cancer at the molecular level. Eur Urol. 2015;67:470-9.

7. Gibbons JA, Ouatas T, Krauwinkel W, et al. Clinical pharmacokinetic studies of enzalutamide. Clin Pharmacokinet. 2015. doi:10.1007/s40262-015-0271-5 (Epub 28 Apr).

8. Gibbons JA, de Vries M, Krauwinkel W, et al. Pharmacokinetic drug interaction studies with enzalutamide. Clin Pharmacokinet. 2015. doi:10.1007/s40262-015-0283-1 (Epub 1 May).

9. Astellas Pharma US, Inc. Xtandi (enzalutamide) capsules prescribing information. Northbrook: Astellas Pharma; 2012. 